\title{
Current Treatments for Delusional Disorder
}

\author{
Mohsen Jalali Roudsari, $M D^{1}$ \\ Jinsoo Chun, Ph.D. ${ }^{1,2}$ \\ Theo C. Manschreck, MD, MPH ${ }^{1,2,3,4, *}$
}

\author{
Address \\ ${ }^{1}$ Laboratory for Clinical and Experimental Psychopathology, Harvard Common- \\ wealth Research Center of Excellence in Clinical Neuroscience and Psychopharma- \\ cological Research, Fall River, MA, USA \\ ${ }^{2}$ Department of Psychiatry, Beth Israel Deaconess Medical Center, Boston, MA, \\ USA \\ ${ }^{3}$ Department of Psychiatry Harvard Medical School, Brockton, MA, USA \\ ${ }^{*}, 4$ Dr. John C. Corrigan Mental Health Center, 49 Hillside Street, Fall River, MA \\ 02720, USA \\ Email: theo.manschreck@state.ma.us
}

Published online: 11 April 2015

C Springer International Publishing AG 2015

This article is part of the Topical Collection on Schizophrenia and Other

Psychotic Disorders

Keywords Clozapine - Cognitive behavioral therapy - Delusions - Depression - First-generation antipsychotics . Second-generation antipsychotics - Neuropsychology · Psychotherapy

\section{Opinion Statement}

Delusional disorders form part of the spectrum of psychotic disorders in psychiatry. They are poorly understood in practically every aspect of their nature, including cause, phenomenology, prevalence, comorbidity, course, treatment, and prognosis. The key symptom in delusional disorders is one or more delusions. A cardinal characteristic of delusional disorder, conviction that one is not mentally ill, contributes complexity to the treatment challenges and profoundly affects the therapeutic relationship. Diagnosis and differential diagnosis are critical tasks. Delusional conditions may arise from many sources and, only when the case conforms to the criteria and is clearly idiopathic-is a primary delusional disorder-can we feel confident that the patient suffers from delusional disorder rather than a secondary delusional condition. Adequate treatment follows principles for the treatment of delusions. First line pharmacological treatment for delusional disorder is antipsychotic medication, both first and second-generation agents, and some evidence suggests that clozapine may be effective in certain cases. The assumption in such a formulation is that the delusion(s) encountered in delusional disorders respond as a class of symptoms found in different disorders. Hence, the approach for failed interventions with these agents may be similar to that proposed for the treatment of schizophrenia or other psychotic disorders. Namely, searching for evidence of failure to take medication, inadequate dosing, a missed diagnosis of a substance disorder, medical condition, or even another psychiatric disorder should be considered. For individuals with delusional 
disorders who are also experiencing comorbid depression or anxiety, the addition of appropriate agents for those symptoms may provide a synergistic strategy for overall effectiveness. Psychotherapy is another important clinical tool. Cognitive behavioral therapy focusing on different aspects of delusions (i.e., anxiety, reasoning biases, faulty logic, etc.) has demonstrated some value for at least short-term improvement. Various techniques may be useful in allying with the patient and supplying means to assist in managing their thinking and approach to circumstances and factors that promote delusion formation and encourage actions taken to respond. A significant problem in applying what we know about delusional disorders to treatment is the limited available evidence. Lack of randomized controlled trials, which form the standard in most clinical disorders, is a major shortcoming. Recent observations of compromised cognitive functioning in working memory, attention, and executive function should be replicated with similar standards. Systematic case studies and large series are useful, and a growing effort to be more rigorous methodologically in all contributions including case registries and longer followup studies is promising. Nevertheless, our knowledge remains less than optimal. These comments apply not only to pharmacological observations but also to those of neuropsychological and psychotherapeutic studies.

\section{Introduction}

Delusional disorders are enigmatic, unusual, and seldom encountered challenges for most clinicians. They are accurately described as uncommon, especially when compared to other psychotic conditions, such as bipolar and schizophrenia disorders. Yet, they are not necessarily rare. Case studies or series of treated individuals diagnosed with this disorder form the foundation of our knowledge about it. However, the frequency of untreated individuals that occurs in the general population remains unclear. Observations of diagnosed cases provide a framework for recognition of these conditions and a source of evidence for how to approach their care.

In this report, we update readers concerning changes in the criteria for this disorder in DSM5 [1], current directions in relevant psychological therapies, and a review of recent contributions on psychopharmacological treatment. At the outset, we must emphasize that the evidence for treatments is limited and suggested strategies are only partially tested.

\section{Description and Modifications of Criteria in DSM-V}

The central feature of delusional disorders is one or more delusions. In earlier definitions, these delusions were restricted to being about possible life experiences, such as being persecuted, having a disease, having an unfaithful partner, etc. This key feature of the disorder must be present for a month. The complementary phenomenological characteristic of delusional disorder is the relative absence of other psychopathology. Additionally, in DSM-V, the clinician is able to rate current symptoms and after a year of the rating can use new criteria to characterize the course of the disorder.

DSM-V has made relatively small changes in the definition of delusional disorders. Addition of bizarreness as a feature that is specified according to a criterion of being "implausible, not understandable, and not derived from ordinary life experiences" is an effort to avoid the unreliability of the concept of bizarreness and to establish a better grasp of the phenomenology 
of delusional experiences in this disorder. Subtypes include erotomanic, grandiose, jealous, persecutory, somatic, mixed, and unspecified. Of these, the persecutory subtype is generally reported to be the most frequent. However, the unusual nature of some forms of somatic delusional illness has attracted attention recently. There has been significant, lay interest in Morgellons' syndrome, a condition characterized by somatic dermatological changes which are claimed to originate from parasitic or other sources. Several publications in the recent literature have dealt with delusional parasitosis or infestation.

DSM-V attempts to clarify the boundaries of delusional disorder and those of body dysmorphic disorder and obsessive-compulsive disorder by noting their phenomenological overlap and the common presence of psychotic variants-emphasizing the need to exclude such cases from the delusional disorder group. Shared delusional disorder is no longer a separate disorder; other specified schizophrenia spectrum and other psychotic disorder may be appropriate diagnoses if the delusional disorder criteria are not met and shared delusions are present. Alternatively, such cases can be diagnosed as delusional disorder if they conform to the criteria.

\section{Psychopharmacological Treatment}

\section{Methods}

A search of PubMed was performed to find available reports on the pharmacological treatment of delusional disorder (DD) published in English-between 2012 and 2014.

Adequate diagnostic accuracy, treatment descriptions or outcome information were among our selection criteria. We identified 24 articles including 15 case reports with 25 cases, one case series of 50 cases and 6 observational studies with 384 cases.

\section{Demographics}

Table 1 provides an overview on the 6 observational studies, the case series and 25 case reports that met the DSM-IV-TR or ICD10 criteria for DD. There was a gender difference with female preponderance in our collection, which was in line with prior studies $[8 \bullet \bullet, 9 \bullet \bullet, 10,11 \bullet \bullet]$. However, a recent follow-up study on gender-related features in DD with 43 patients reported no gender difference

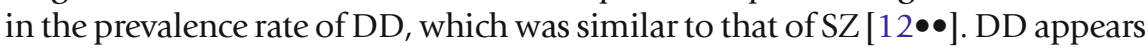
to be diagnosed more frequently in middle-aged or elderly individuals. They become ill much later than in schizophrenia [6]. The mean age of onset in recent reports (Table 1) was older than 45 years. Further, in our summary of 25 individuals [13-27], women became ill significantly later than men which is consistent with other recent studies $[2 \bullet \bullet, 7 \bullet \bullet, 12 \bullet \bullet]$ and some previous ones [10]. Since DD appears to be a disorder mostly seen in later stages of life, physicians and caregivers should be cautious about medication side effects and drug-drug interactions when it comes to pharmacological treatment. 


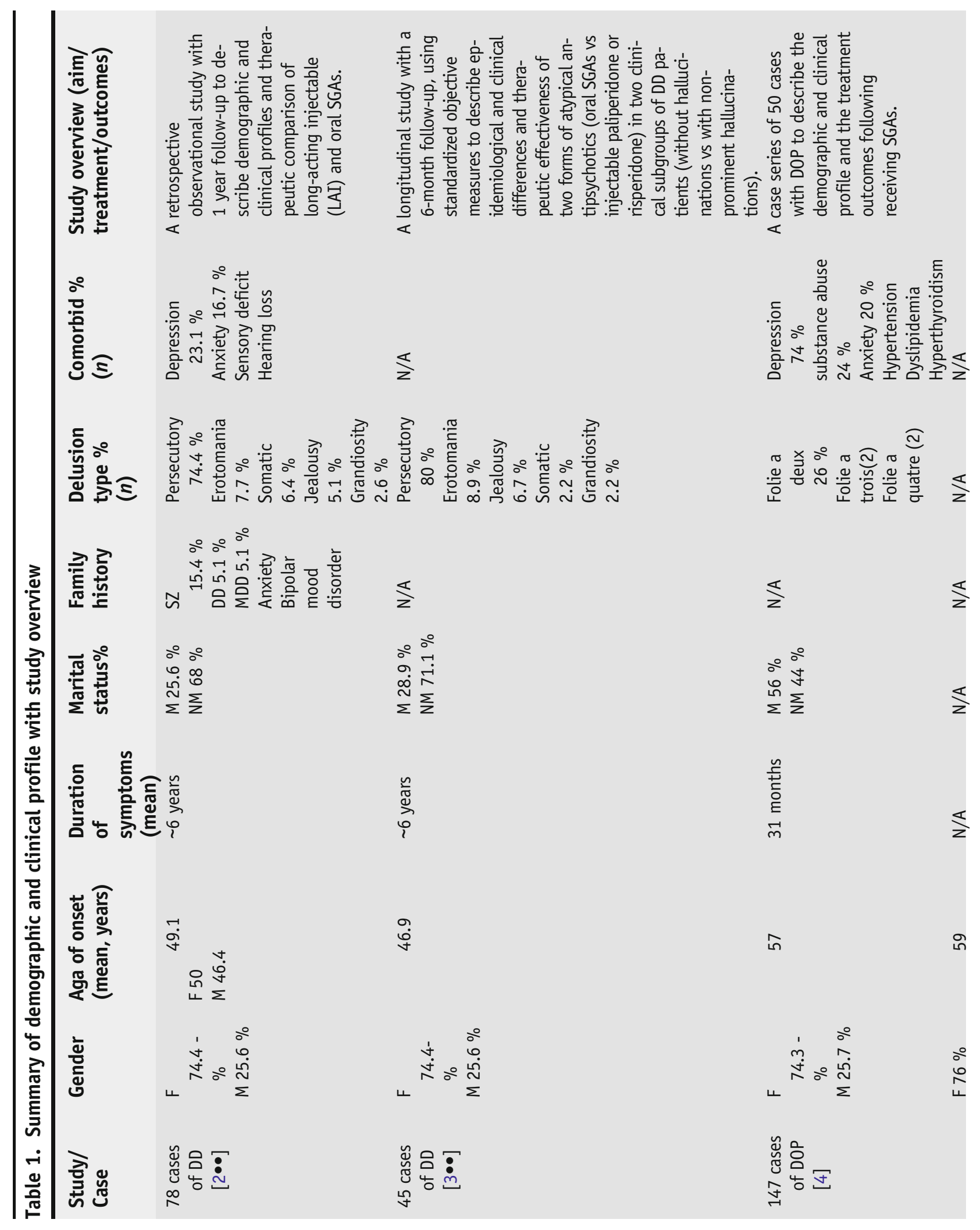




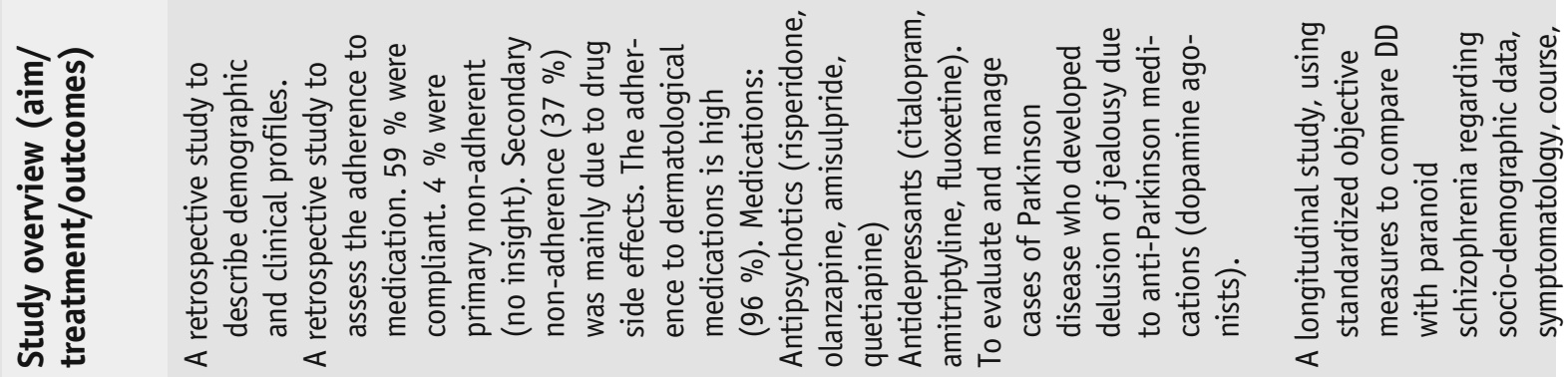

웅
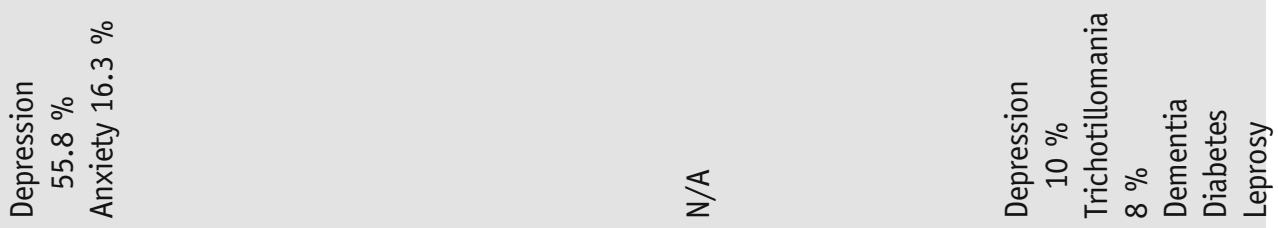

흔ำำ

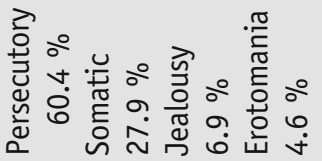

$\frac{1}{z}$

$\frac{1}{2}$

를 중

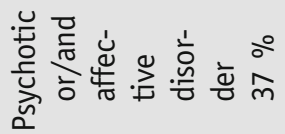

$\frac{1}{z}$

$\frac{1}{z}$

焉

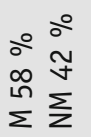

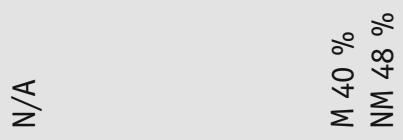

亭

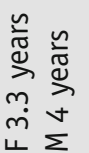

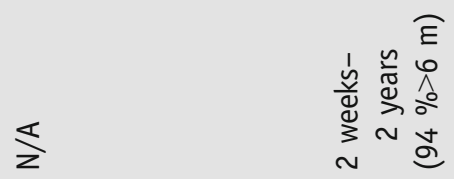

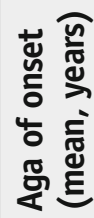

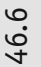

$\lesssim$

$\stackrel{1}{2}$
$\stackrel{2}{\circ}$
$\infty$
$\infty$

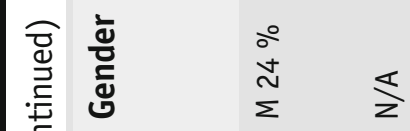

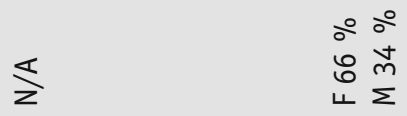

离

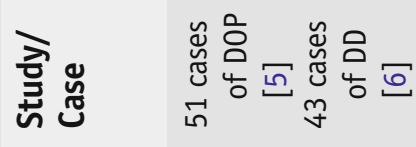

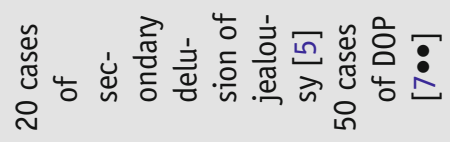



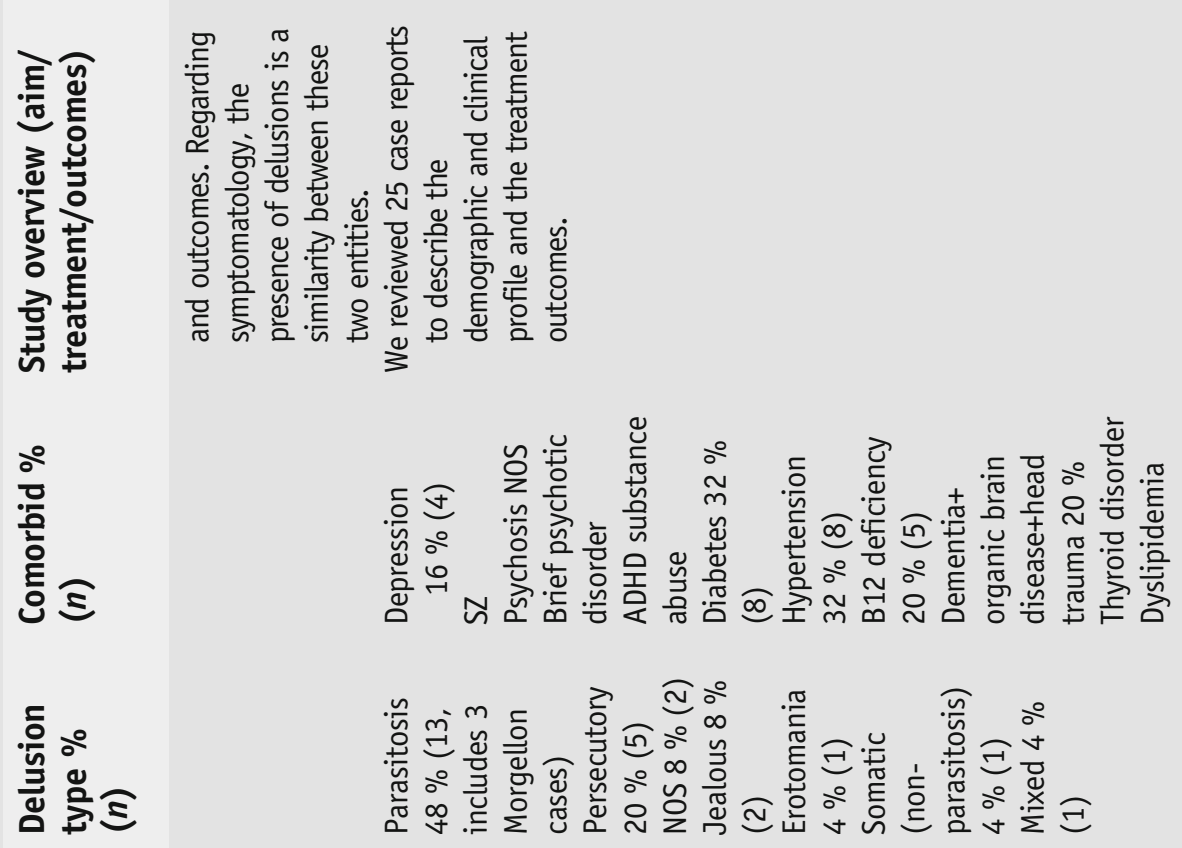

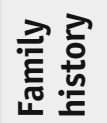

$\frac{\pi}{z}$

焉旁

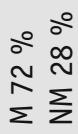

总

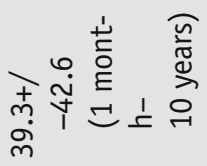

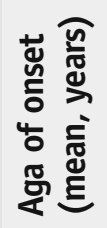

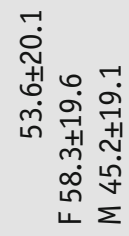

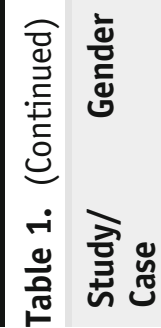
ㅇํㅇำ
to

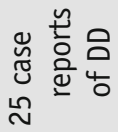

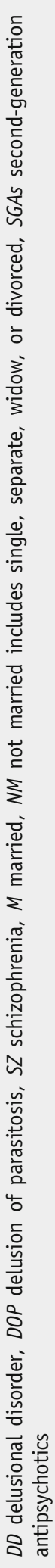


DD appears to be a disorder with relatively high diagnostic stability over time. A follow-up study [6] showed a stable diagnosis over more than 10 years in almost $79 \%$ of the patients with DD. Women showed a higher diagnostic stability than men, while men tended more frequently to be re-diagnosed with

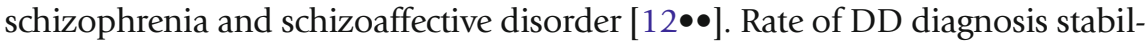
ity in a work by Salvatore et al. [28] was $72.7 \%$.

The most frequent type of delusional disorder among our 25 cases was delusion of parasitosis (48 \%) including 3 cases of Morgellons. Persecutory type was the next common type with $20 \%$. Among the 6 observational studies, we found three exclusively addressed cases of delusion of parasitosis with the sample of 198 cases. The case series of 50 cases also studied delusion of parasitosis (Table 1). Regarding the most frequent types of DD, our reports are not consistent with other evidence. In two recent longitudinal observational studies on patients with DSM-IV-TR diagnosis DD with sample of 78 and 45

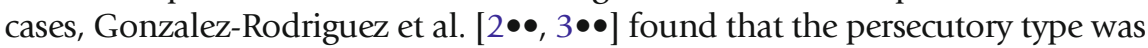
the most frequent type with 80 and $74.4 \%$, respectively, which is consistent with other observations $[9 \bullet \bullet, 10,12 \bullet \bullet]$. Regarding the correlation between the

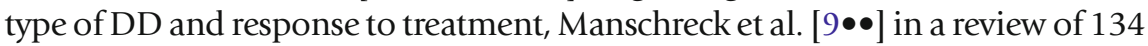
DD patients reported that delusion of a somatic type were more responsive to antipsychotics than other delusion types.

Diagnosis of comorbid conditions is crucial in DD, since these conditions may influence response to treatment and may cause clinicians to alter treatment approaches. The majority of studies on DD reported depression or depressive symptoms as the most frequent comorbidities $[2 \bullet \bullet, 4,6,7 \bullet \bullet, 9 \bullet \bullet, 11 \bullet \bullet]$. In 2011, de Portugal et al. [11••] evaluated 86 patients with DSM-IV-DD for psychiatric comorbid conditions. Forty six percent of this sample had at least one additional lifetime psychiatric diagnosis. The diagnosis of depressive disorders (32.6\%) was the most common, followed by anxiety disorders (14\%). Individuals with Axis I comorbidities tended to show more conditions or symptoms such as cluster C personality psychopathology, somatic delusions, olfactory and gustatory hallucinations, and suicide risk as well as emotional dysregulation. However, working memory deficit was worse in DD patients without psychiatric comorbidity. These findings suggested that emotional and cognitive aspects of the illness should be considered and may need to be monitored in DD treatments.

\section{Antipsychotics}

The first step in treatment of DD is to make an accurate diagnosis, distinguishing between primary and secondary DD through careful differential diagnosis. Secondary DD may be managed by treating the underlying causes. For example, in a study on 20 patients with Parkinson disease who developed delusions of jealousy due to dopamine agonist medications, Perugi et al. [29] showed that withdrawal of dopamine medications led to complete remission and reduction of doses to partial remission of psychotic symptoms. Investigation for possible comorbid conditions is also essential, including both medical and psychiatric diseases as they may influence response to treatment and may alter treatment approach.

Although there is a lack of randomized placebo-controlled studies on DD treatment, literature suggests optimistic outcomes with antipsychotics with a 
high positive response rate-remission or partial remission $[3 \bullet \bullet, 7 \bullet \bullet, 8 \bullet \bullet, 9 \bullet \bullet$, $10,30,31]$. For example, a 6 -month follow-up study on 45 DD inpatients that compared the efficacy of oral second-generation antipsychotic (SGAs) and long acting risperidone/paliperidone demonstrated lower Positive and Negative

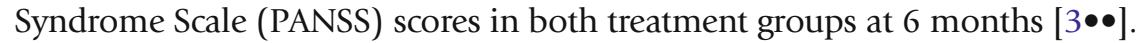

Regarding the frequency of use of first-generation antipsychotic (FGA) and

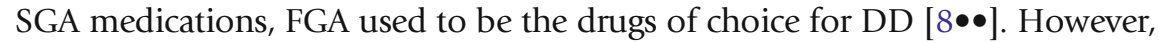
the introduction of atypical antipsychotics and the experience that pimozide is not as specific as considered earlier, SGAs have been used more frequently. In our review, studies (Table 1) with available data on the type of antipsychotics addressed SGAs $[2 \bullet \bullet, 3 \bullet \bullet, 5,7 \bullet \bullet]$. In addition, more than $80 \%$ of 25 case reports received antipsychotics, mostly SGAs (SGA $64 \%$, FGA $12 \%$ ), with risperidone as the most frequent medication (24\%) followed by olanzapine, quetiapine, and FGA (Table 2).

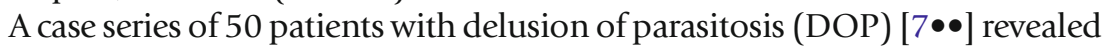
that all 45 patients on antipsychotics received SGAs. Antipsychotic treatment was associated with a high positive response rate (recovery $64.4 \%$ or partial improvement $28.8 \%$ ), with risperidone as the most frequent medication followed by olanzapine (Table 3). Among SGAs, risperidone appears to be the most frequent antipsychotic that has been used in DD treatment. There is no controlled trial to compare treatment efficacy between FGAs and SGAs. Two early reports showed no difference $[9 \bullet \bullet, 31]$. One study reported that risperidone showed the best response rate (full and partial remissions) among antipsychotics followed by pimozide [10]. A comprehensive review of DOP reported that if "full remission" only is considered, FGAs (mostly pimozide) were associated with a higher response rate compared to SGAs (52 vs $37 \%$ ) [30]. There had been a similar range of success with pimozide in somatic delusional disorder $[8 \bullet \bullet, 9 \bullet \bullet]$.

Randomized controlled trials with systematic outcome measures are required to address treatment efficacy of different pharmacological agents for DD. Factors such as heterogeneity of study designs, small sample sizes, adherence, and related problems (i.e., lack of insight, medication side effects and inadequate trials) have limited progress. Side effect features as a criterion for selecting medications should be considered carefully. The higher average age of onset in DD compared to other Axis I psychotic disorders may be associated with greater vulnerability to adverse medication effects. The age of the patients, the presence of comorbid conditions, and drug interactions also should be

Table 2. Antipsychotics and outcomes-25 case reports

\begin{tabular}{llll}
\hline Antipsychotic & Recovery & Partial improvement & No improvement \\
SGA (17) & 8 & 4 & 5 \\
FGA (3) & 3 & 0 & 0 \\
Both (SGA+FGA) (1) & 1 & 0 & 0 \\
Antidepressant (2) & 1 & 1 & 0 \\
Psychotherapy (1) & 1 & 0 & 0 \\
Non (1) & 1 & 0 & 0 \\
Total (25) & 15 & 5 & 5
\end{tabular}




\begin{tabular}{lccc}
\hline Table 3. Antipsychotics and outcomes-Bhatia et al. [7••] & Partial improvement & No improvement \\
\hline Antipsychotic & Recovery & 3 & 1 \\
Risperidone (12) & 8 & 3 & 1 \\
Olanzapine (9) & 5 & 3 & 1 \\
Amisulpride (7) & 3 & 2 & 0 \\
Quetiapine (5) & 3 & 1 & 0 \\
Aripiprazole (5) & 4 & 1 & 0 \\
Paliperidone (5) & 4 & 0 & 0 \\
Iloperidone (2) & 2 & 0 & 0 \\
Fluoxetine (5) & 5 & 13 & 3 \\
Total (50) & 34 & &
\end{tabular}

considered in selecting agents. Pimozide seems no longer to be the first choice in treatment of DD. This might be mostly due to the concern about the side effects such as extrapyramidal symptoms, dose-dependent QTc interval prolongation, and drug-drug interactions. However, pimozide might be considered for younger patients, as a monotherapy, in low dose, and with QTc monitoring.

Similarly, there are SGAs side effect concerns. Olanzapine and quetiapine are both associated with significantly greater weight gain and risks of glucose and lipid dysregulation compared to other antipsychotics [32-36]. Quetiapine is also associated with QTc prolongation. Quetiapine, when combined with other drugs with a QTc risk such as citalopram, is also a concern. Risperidone, amisulpride, aripiprazole, and ziprasidone can be considered as slightly more tolerable agents. Sexual dysfunction and movement disorders are the important risperidone side effects but can be manageable [37]. Aripiprazole is more tolerable in terms of movement disturbances, metabolic issues, prolactin increases, and QTc prolongation [38]. Amisulpride induces less weight gain than risperidone and olanzapine. Ziprasidone has probably the lowest tendency of any SGA to induce weight gain and associated metabolic problems. However, it is associated with QTc issues [39]. Osser et al. [40] in a recent guideline on treatment of positive symptoms of schizophrenia, including delusion, emphasized that the FGAs and SGAs such as olanzapine, clozapine, and quetiapine should not be considered as the first line agents for schizophrenia, due to their side effect profiles. This may have relevance to DD treatment strategy.

Despite limited data regarding effective antipsychotic dosing for DD treatment, it is notable that the initial dosages in DD studies were lower than the minimum dosage recommended for the treatment of schizophrenia.

\section{Combination Therapy}

Selective serotonin reuptake inhibitors (SSRIs) and serotonin norepinephrine reuptake inhibitors (SNRIs) are the medications often used in DD treatment. Despite some common side effects (i.e., GI disturbance, sexual dysfunction, weight gain or loss, insomnia, restlessness, and agitation), relatively low side effect impact has been reported. However, possible medication interaction is among the concerns. For example, citalopram and escitalopram are associated with dose-dependent QTc prolongation. Similarly, clinicians need to be cautious when using SSRIs or SNRIs with patients who have QTc condition. 
Combining different psychotropic agents is a frequent approach in DD treatment. Forty four percent (11) of our selected 25 case reports (Table 2) received combination therapy with an antidepressant, valproate, or psychotherapy, which yielded $>90 \%$ positive response (recovery or partial improvement). Three cases received antidepressant, valproate, or psychotherapy alone, with each achieving positive response. As noted, depression and anxiety symptoms are the most frequent comorbid conditions with DD. A recent review study on the affective symptoms in DD noted relatively high antidepressant efficacy either as monotherapy or in combination with antipsychotics $[41 \bullet \bullet]$. Another case study supported such an interpretation in that $10 \%$ of DOP patients with comorbid depression fully remitted with fluoxetine monotherapy $[7 \bullet \bullet$ ] (Table 3 ).

\section{Long-Acting Injectables}

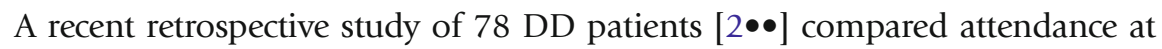
psychiatry follow-up visits in 3 treatment groups: long-acting injectables (LAIs), oral risperidone, or other oral SGAs. At 1 year, higher treatment maintenance rates and less antidepressant and benzodiazepine usage was found in LAIs group compared to oral SGAs. The same researchers performed a 6-month follow-up study on 45 patients with DD patients [3••] to compare treatment effectiveness of LAIs (paliperidone palmitate or risperidone) with oral risperidone and other oral SGAs. Using several measures to assess the outcomes such as PANSS, Hamilton Rating Scale for Depression, the Personal and Social Performance Scale, they found that the LAIs group showed a trend toward lower scores in PANSS positive symptoms compared to the oral antipsychotics groups and significantly lower scores in PANSS negative symptoms. These findings of greater improvement in negative symptoms may be attributed to the nature of high DD comorbidity with depression, which may manifest as psychomotor retardation.

\section{Neuropsychological Insights Related to Treatment for Delusional Disorder}

The focus of neuropsychological approach in the treatment of DD has been centered in the formation of delusional beliefs and their maintenance based on the cognitive models of human brain function. Although cognitive impairment among patients with DD has been reported to be less than that of patients diagnosed with schizophrenia [42], deficits in cognitive domains such as executive function and working memory have been reported [43, $44 \bullet \bullet$ ]. Studies with patients with delusions from Freeman's research group have provided further details of six core features of delusions in relation to working memory and/or executive deficits identifying them as targets for cognitive behavioral treatment of delusions: worry, negative beliefs about self, interpersonal sensitivity, sleep disturbances, anomalous internal experience, and reasoning biases [45••] (see "Cognitive behavioral therapy (CBT) for delusions"). 
A recent neuroimaging study $[44 \bullet \bullet]$ demonstrated working memory deficits among DD using an n-back task to measure immediate and recent recall capacity. During the task performance, less activation over the left dorsolateral prefrontal cortex and more prominent neural activation over the bilateral superior temporal gyrus, left middle and temporal gyrus, bilateral posterior cingulate gyrus, right amygdala, and bilateral fusiform gyrus have been observed, which was distinct from healthy controls. However, the proposal that such dysfunctional prefrontal, temporal, and limbic regions among DD are characteristic neural deficits should be interpreted with caution not only because of the small sample size $\left(\mathrm{N}_{\mathrm{DD}}=9\right.$, $\mathrm{N}_{\text {controls }}=9$ ) but also because of the lack of distinguishing findings between DD patients and schizophrenia. Another study [46••] examined the contributing cognitive factors to the occurrence of reasoning bias, specifically jumping to conclusions (JTC) among patients with delusions. The study participants were patients diagnosed with schizophrenia spectrum disorders. Patients with JTC bias showed more pronounced deficits in working memory manipulation (letter-number sequencing task) rather than working memory span (digit span forward). Such results indicate executive deficits in organizing and holding information that has been already encoded in working memory. The authors suggested that interventions carefully designed to employ minimal cognitive resources as well as other techniques to compensate for such limited cognitive resources may improve JTC reasoning bias. Such executive and working memory deficits associated with delusion were further supported by Ibanez-Casas [43] study of DD. This large study a sample size with DD patients $\left(\mathrm{N}_{\mathrm{DD}}=86\right.$, $\mathrm{N}_{\text {controls }}=343$ ) tested executive and memory deficits among patients. Standardized cognitive tests results demonstrated significant deficits in executive function (i.e., fewer categories in WCST), impulsivity (i.e., more errors in Stroop task), and working memory (i.e., reduced immediate recall in a computerized verbal learning test) among DD patients even when age, education, and premorbid IQ were controlled. Reduced working memory during paranoia has been demonstrated in a randomized, placebo-

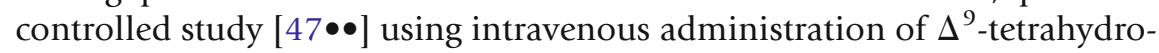
cannabinol (THC; $1.5 \mathrm{mg}$ dose, $99.5 \%$ purity) among people with paranoid ideation. However, the changes in working memory induced by THC did not lead to paranoia, while the increases in negative affect and anomalous experiences fully accounted for the added paranoia. It is notable that group that received THC and brief cognitive awareness session did not experience reduced paranoid thoughts, which in contrast, may have increased sensitivity to paranoid thoughts.

Cognitive Behavioral Therapy for Delusions

Cognitive behavioral treatment of delusion, as a core symptom of DD, has been developed to identify and improve data gathering bias, interpersonal sensitivity [48], reasoning style [49], worry [50], and insomnia [51] as short term period of treatment. A randomized experimental investigation [52] reported that data gathering could be improved for a short term period training. A single training consisted of 3 tasks (object identification, picture interpretation, and illusion) 
designed to depict reasoning biases and ways to correct them. The delusional groups showed significant improvement in data gathering (i.e., in JTC task, more beads observed before decision making), although more severe form of data gathering bias (the number of participants asking two or less beads before decision making) did not change. Since the authors did not provide other data including symptom severity, affective state, and other cognitive domains (i.e., working memory, executive function), the findings should be interpreted with caution. Follow-up studies with longer intervention periods are still warranted. A pilot trial of cognitive behavioral therapy (CBT) [48] reported promising results that short-term CBT targeting interpersonal sensitivity (CBT-IPS) could reduce persecutory delusions. They performed six sessions of CBT during the 12-week study period. The intervention consisted of basic CBT principles (i.e., collaborative working, behavioral tests, reduction of risky behaviors, attention switching, and feedback on therapy) with the emphasis on experiential learning. Findings of reduced interpersonal sensitivity $(d=0.96)$ and persecutory delusion $(d=0.94-3.26)$ call for more randomized, controlled clinical trials to further establish the efficacy of CBT-IPS in treatment settings. A replication

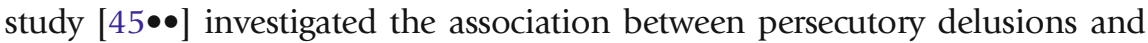
specific patterns of the dual reasoning processes (rational and experiential reasoning), which is higher intuitive or experiential reasoning and lower levels of analytic reasoning among both currently delusional patients $(n=30)$ and large non-clinical groups $(n=1000)$. Using self-report measures of persecutory delusion, the previous findings were not fully replicated in that clinical paranoia was associated with less analytic thinking as well as less experiential reasoning style. However, higher rational engagement was associated with less paranoia among non-clinical groups, while higher rational engagement was associated with higher degrees of paranoia in the patient group. Studies with clinician's rating as well as cognitive performance measures (i.e., MATRICS) are warranted to clarify the role of dual reasoning processes in the occurrence of persecutory delusions. Hepworth et al. [50] performed a pilot study to test the efficacy of new brief treatment technique, Emotional Processing and Metacognitive Awareness Intervention (EPMA) with twelve patients with delusions. Three 1-hour interventions were provided, focusing on increasing access to emotional experience, reducing verbal-linguistic processing (i.e., describing current experience as it is in a non-judgmental manner by using experiential focus), metacognitive awareness training (i.e., labeling and writing down thoughts, feelings, sensations, images, and memories to recognize that they are thoughts rather than facts) to take a "decentered" perspective and enhance metacognitive insight, accepting their experience without judgments. The gist of EPMA is to facilitate emotional processing of upsetting delusional experience in order to block the cycle of worry-ruminative appraisal of delusion-related events. EPMA in this study led to a significant reduction in delusional distress measured by Psychotic Symptoms Rating Scale-Delusions (effect size $=1.03$ ), which remained stable at 1-month follow-up. Although replication with a randomized, controlled design using larger samples is warranted, this study is intriguing as it appears to demonstrate that talking about emotional experience out loud may become a clinically useful intervention strategy in such cases. A pilot study, non-blinded, uncontrolled, by Myers et al. [51] provided an excellent example of "interventionist-causal model" on single symptom, insomnia. A four-session standard CBT consisted of psychoeducation about sleep difficulty, assessment 
of sleep disturbance, and goal setting (session I), sleep diary, sleep hygiene (i.e., tackling sleep problems, lifestyle factors affecting sleep disturbance, bedroom conditions), stimulus control (i.e., associating bed with sleep; session II), relaxation, training to overcome sleep-related worries (session III), followed by sleep review and relapse prevention (session IV). This CBT intervention targeting sleep disturbance was associated with a significant reduction in persecutory delusions (effect size $=1.07$ ) as well as insomnia (effect size $=2.64$ ). The intervention effect was maintained through a 1-month follow-up period. The promising results of CBT in the reviewed studies warrant more blind and controlled studies with longer periods of training and follow-up to determine the maintenance of the treatment effects.

\section{Conclusion}

- Accurate diagnosis of DD, an idiopathic condition, and exclusion of cases with delusions with known causes, are essential to successful treatment. DD is far less common than the latter, secondary forms of disorder.

- Comorbid disorders are frequent and influence the presentation of DD. They should be addressed therapeutically for optimal results.

- Understanding that the DD patient is convinced that mental illness is not the source of distress is the fundamental challenge for effective treatment.

- The delusion(s) of DD respond to treatment, meaning they may become less complained of, less focused upon by the patient, and less likely to lead to actions inspired by delusional concerns. They can recur and this feature is a critical monitoring challenge.

- Various treatments can target delusion(s) in DD: psychopharmacological and psychological therapies have been applied and have demonstrated impact.

- First and second-generation antipsychotic agents have shown positive results; pimozide and clozapine also have value despite side effects and limited evidence. Long-acting antipsychotic agents have shown impact as well.

- While there are advantages associated with various agents, the key strategy is to select agents based on side effect risks, ease of administration, and acceptability to the patient.

- Neuropsychological observations have pointed to cognitive compromise in inductive reasoning, for example, that may perpetuate delusional thinking and has inspired specific CBT strategies.

- Emotional responses, such as worry and interpersonal sensitivity, have also been singled out for focused psychological treatments.

- While these developments are promising, the evidence fails to match the methodological standards usually required in clinical psychiatric therapeutics. 


\section{Compliance with Ethics Guidelines}

\section{Conflict of Interest}

Mohsen Jalali Roudsari, Jinsoo Chun, and Theo C. Manschreck declare that they have no conflict of interest.

Human and Animal Rights and Informed Consent

This article does not contain any studies with human or animal subjects performed by any of the authors.

\section{References}

Papers of particular interest, published recently, have been highlighted as:

$\bullet \quad$ Of major importance

1. Association AP. Diagnostic and statistical manual of mental disorders (5th ed.). Washington, DC. 2013.

2.•• Gonzalez-Rodriguez A, Molina-Andreu O, Imaz Gurrutxaga ML, Catalan Campos R, Bernardo Arroyo $\mathrm{M}$. A descriptive retrospective study of the treatment and outpatient service use in a clinical group of delusional disorder patients. Rev Psiquiatr Salud Ment. 2014;7(2):64-71. doi:10.1016/j.rpsm.2013.01.004. A longitudinal retrospective study with one-year follow-up, to describe demographic and clinical features in DD as well as to compare attendance at psychiatry follow-up visit in 3 treatment groups: LAIs, oral risperidone, or other oral SGAs. $\mathrm{N}=78$. At one year, higher treatment maintenance rates and less antidepressant and benzodiazepine usage were found in LAIs group compared to oral SGAs.

3.• Gonzalez-Rodriguez A, Molina-Andreu O, Penades R, Bernardo M, Catalan R. Effectiveness of long-acting injectable antipsychotics in delusional disorders with nonprominent hallucinations and without hallucinations. Int Clin Psychopharmacol. 2014;29(3):177-80. doi:10.1097/yic.0000000000000020.

A longitudinal observational study on delusional disorder with 6-month follow-up- $\mathrm{N}=45$ - to describe demographic and clinical features in DD as well as to compare treatment outcomes between two forms of atypical antipsychotics (oral antipsychotics vs injectable paliperidone or risperidone) in two clinical subgroups of DD patients (without hallucinations vs with non-prominent hallucinations). This study is among a few studies on DD which use objective measures such as PANS S, Hamilton Rating Scale for Depression, the Personal and Social Performance Scale and drug compliance assessment to assess the outcomes. The LAIs group showed a trend toward lower scores in PANSS positive symptoms compared to the oral antipsychotics groups, and significantly lower scores in PANSS negative symptoms.

4. Foster AA, Hylwa SA, Bury JE, Davis MD, Pittelkow MR, Bostwick JM. Delusional infestation: clinical presentation in 147 patients seen at Mayo Clinic. J Am Acad Dermatol. 2012;67(4):673 e1-10. doi:10.1016/j.jaad. 2011.12.012.
5. Ahmed A, Bewley A. Delusional infestation and patient adherence to treatment: an observational study. Br J Dermatol. 2013;169(3):607-10. doi:10.1111/bjd. 12392.

6. Marneros A, Pillmann F, Wustmann T. Delusional disorders-are they simply paranoid schizophrenia? Schizophr Bull. 2012;38(3):561-8. doi:10.1093/ schbul/sbq125.

7.• Bhatia MS, Jhanjee A, Srivastava S. Delusional infestation: a clinical profile. Asian J Psychiatr. 2013;6(2):124-7. doi:10.1016/j.ajp.2012.09.008. Is a case series of 50 cases with delusion of parasitosis and described the demographic and clinical features as well as pharmacological treatment outcomes. 45 patients received SGAs. Antipsychotic treatment was associated with a high positive response rate-full or partial remission, with risperidone as the most frequent medication followed by olanzapine. $10 \%$ of DOP patients had comorbid depression and fully remitted with fluoxetine monotherapy.

8.• Munro A, Mok H. An overview of treatment in paranoia/delusional disorder. Can J Psychiatry. 1995;40(10):616-22.

A review of 209 reports of cases with DD published between 1961 and 1994, describing the demographic and clinical features as well as pharmacological treatment outcomes. All the patients received neuroleptics. If treated adequately, DD is a disorder with good prognosis. More than $80 \%$ of patients showed partial or complete remission. Pimozide was suggested as the first line treatment.

9.• Manschreck TC, Khan NL. Recent advances in the treatment of delusional disorder. Can J Psychiatry. 2006;51(2):114-9.

A review of 134 cases with DD published between 1995 and 2004, described the demographic and clinical features as well as pharmacological treatment outcomes. Antipsychotic therapy was associated with high rate of positive results-full or partial remission. There was no significant difference between FGAs and SGAs in outcomes. Pimozide was associated with greater rate of improvement in somatic delusion compared to other types of delusions. 
10. Grover S, Biswas P, Avasthi A. Delusional disorder: study from North India. Psychiatry Clin Neurosci. 2007;61(5):462-70. doi:10.1111/j.1440-1819.2007. 01694.x.

11.• de Portugal E, Martinez C, Gonzalez N, del Amo V, Haro JM, Cervilla JA. Clinical and cognitive correlates of psychiatric comorbidity in delusional disorder outpatients. Aust N Z J Psychiatry. 2011;45(5):416-25. doi:10.3109/00048674.2010. 551279.

Used objective measured to investigate the prevalence, and the possible correlation between psychiatric comorbidities and clinical and cognitive features in DD $(n=86)$. Depression and anxiety were the most common comorbidities. Those with psychiatric comorbidity showed greater emotion-related psychopathology and those without psychiatric comorbidity were associated with more cognitive disturbances.

12.• Wustmann T, Pillmann F, Marneros A. Gender-related features of persistent delusional disorders. Eur Arch Psychiatry Clin Neurosci. 2011;261(1):29-36. doi:10. 1007/s00406-010-0130-1.

As part of the Halle Delusional Syndrome Study (HADESStudy), described gender-related features of DD in 43 inpatients DD. There was no gender difference in the prevalence rate of DD. Women became ill significantly later than men. They also showed a higher diagnosis stability than men, while men tended more frequently to be re-diagnosed with schizophrenia and schizoaffective disorder.

13. Rajkumar RP. Supersensitivity psychosis and its response to asenapine in a patient with delusional disorder. Case Rep Psychiatry. 2014;2014:215732. doi:10. 1155/2014/215732.

14. Noel J, Krishnadas R, Gopalakrishnan R, Kuruvilla A. Delusional disorder: an unusual presentation. Ind J Psychol Med. 2014;36(4):444-6. doi:10.4103/02537176.140757.

15. Ukai K, Kimura H, Arao M, Aleksic B, Yamauchi A, Ishihara $\mathrm{R}$, et al. Effectiveness of low-dose milnacipran for a patient suffering from pain disorder with delusional disorder (somatic type) in the orofacial region. Psychogeriatrics. 2013;13(2):99-102. doi:10.1111/j. 1479-8301.2012.00430.x.

16. Shah R, Faruqui RA. Delusional jealousy and person directed hostility: 5-year follow-up of a patient after anoxic brain injury. Brain Inj. 2013;27(13-14):171922. doi:10.3109/02699052.2013.831129.

17. Prakash O, Garg A, Sinha P. Low-dose quetiapine for the treatment of delusional disorder in a patient with von Hippel-Lindau disease. J Neuropsychiatry Clin Neurosci. 2013;25(3):E54. doi:10.1176/appi. neuropsych.12080192.

18. Ozten E, Tufan AE, Cerit C, Sayar GH, Ulubil IY. Delusional parasitosis with hyperthyroidism in an elderly woman: a case report. J Med Case Rep. 2013;7(1):17. doi:10.1186/1752-1947-7-17.

19. Mews MR, Quante A. Comparative efficacy and acceptability of existing pharmacotherapies for delusional disorder: a retrospective case series and review of the literature. J Clin Psychopharmacol. 2013;33(4):512-9. doi:10.1097/JCP. ob013e3182905796.

20. Huang WL, Chang LR. Aripiprazole in the treatment of delusional parasitosis with ocular and dermatologic presentations. J Clin Psychopharmacol. 2013;33(2):272-3. doi:10.1097/JCP. 0b013e3182856850.

21. De Berardis D, Serroni N, Marini S, Rapini G, Valchera A, Fornaro M, et al. Successful ziprasidone monotherapy in a case of delusional parasitosis: a one-year followup. Case Rep Psychiatry. 2013;2013:913248. doi:10.1155/2013/913248.

22. Prakash J, Shashikumar R, Bhat PS, Srivastava K, Nath S, Rajendran A. Delusional parasitosis: worms of the mind. Ind Psychiatry J. 2012;21(1):72-4. doi:10.4103/ 0972-6748.110958.

23. Lew-Starowicz M. Shared psychotic disorder with sexual delusions. Arch Sex Behav. 2012;41(6):1515-20. doi:10.1007/s10508-012-9992-9.

24. Kansal NK, Chawla O, Singh GP. Treatment of delusional infestation with olanzapine. Indian J Psychol Med. 2012;34(3):297-8. doi:10.4103/0253-7176. 106043.

25. Contreras-Ferrer P, de Paz NM, Cejas-Mendez MR, Rodriguez-Martin M, Souto R, Bustinduy MG.

Ziprasidone in the treatment of delusional parasitosis. Case Rep Dermatol. 2012;4(2):150-3.

26. Buscarino M, Saal J, Young JL. Delusional parasitosis in a female treated with mixed amphetamine salts: a case report and literature review. Case Rep Psychiatry. 2012;2012:624235. doi:10.1155/2012/624235.

27. Altunay IK, Ates B, Mercan S, Demirci GT, Kayaoglu S. Variable clinical presentations of secondary delusional infestation: an experience of six cases from a psychodermatology clinic. Int J Psychiatry Med. 2012;44(4):335-50.

28. Salvatore P, Baldessarini RJ, Tohen M, Khalsa HM, Sanchez-Toledo JP, Zarate Jr CA, et al. McLean-Harvard International First-Episode Project: two-year stability of DSM-IV diagnoses in 500 first-episode psychotic disorder patients. J Clin Psychiatry. 2009;70(4):458-66.

29. Perugi G, Poletti M, Logi C, Berti C, Romano A, Del Dotto $\mathrm{P}$, et al. Diagnosis, assessment and management of delusional jealousy in Parkinson's disease with and without dementia. Neurol Sci. 2013;34(9):1537-41. doi:10.1007/s10072-012-1276-6.

30. Freudenmann RW, Lepping P. Delusional infestation. Clin Microbiol Rev. 2009;22(4):690-732. doi:10. 1128/CMR. 00018-09.

31. Herbel BL, Stelmach H. Involuntary medication treatment for competency restoration of 22 defendants with delusional disorder. J Am Acad Psychiatry Law. 2007;35(1):47-59.

32. McEvoy JP, Lieberman JA, Perkins DO, Hamer RM, Gu $\mathrm{H}$, Lazarus A, et al. Efficacy and tolerability of olanzapine, quetiapine, and risperidone in the 
treatment of early psychosis: a randomized, doubleblind 52-week comparison. Am J Psychiatry. 2007;164(7):1050-60. doi:10.1176/ajp.2007.164.7. 1050 .

33. Fernandez-Egea E, Miller B, Garcia-Rizo C, Bernardo M, Kirkpatrick B. Metabolic effects of olanzapine in patients with newly diagnosed psychosis. J Clin Psychopharmacol. 2011;31(2):154-9. doi:10.1097/ JCP.0b013e31820fcea3.

34. Robinson DG, Woerner MG, Napolitano B, Patel RC, Sevy SM, Gunduz-Bruce H, et al. Randomized comparison of olanzapine versus risperidone for the treatment of first-episode schizophrenia: 4-month outcomes. Am J Psychiatry. 2006;163(12):2096-102. doi:10.1176/appi.ajp.163.12.2096.

35. Zipursky RB, Gu H, Green AI, Perkins DO, Tohen MF, McEvoy JP, et al. Course and predictors of weight gain in people with first-episode psychosis treated with olanzapine or haloperidol. Brit J Psychiatry: J Mental Sci. 2005;187:537-43. doi:10.1192/bjp.187.6.537.

36. Patel JK, Buckley PF, Woolson S, Hamer RM, McEvoy JP, Perkins DO, et al. Metabolic profiles of secondgeneration antipsychotics in early psychosis: findings from the CAFE study. Schizophr Res. 2009;111(13):9-16. doi:10.1016/j.schres.2009.03.025.

37. Malik P, Kemmler G, Hummer M, Riecher-Roessler A, Kahn RS, Fleischhacker WW. Sexual dysfunction in first-episode schizophrenia patients: results from European First Episode Schizophrenia Trial. J Clin Psychopharmacol. 2011;31(3):274-80. doi:10.1097/ JCP.0b013e3182199bcc.

38. Chung AK, Chua SE. Effects on prolongation of Bazett's corrected QT interval of seven second-generation antipsychotics in the treatment of schizophrenia: a metaanalysis. J Psychopharmacol (Oxford, England). 2011;25(5):646-66. doi:10.1177/ 0269881110376685.

39. Komossa K, Rummel-Kluge C, Hunger H, Schwarz S, Bhoopathi PS, Kissling W, et al. Ziprasidone versus other atypical antipsychotics for schizophrenia. Cochrane Database Syst Rev. 2009;4:Cd006627. doi:10.1002/14651858.CD006627.pub2.

40. Osser DN, Roudsari MJ, Manschreck T. The psychopharmacology algorithm project at the Harvard South Shore Program: an update on schizophrenia. Harvard Rev Psychiatry. 2013;21(1):18-40. doi:10.1097/HRP. 0b013e31827fd915.

41.• González-Rodríguez A, Molina-Andreu O, Odriozola VN, Ferrer CG, Penadés R, Catalán R. Delusional disorder: an overview of affective symptoms and antidepressant use. Eur J Psychiat. 2013;27(4):265-76. doi:10.4321/S0213-61632013000400005.

Is a review of literature to describe the frequency of affective symptoms and antidepressant use in DD (34 related articles published between 1980 to 2012). Authors reported a high frequency of depressive symptoms in DD, but a low antidepressant usage rate. They noted relatively high antidepressant efficacy either as monotherapy or in combination with antipsychotics. They suggested that antidepressant may be useful in treatment of DD with or without comorbid depression. The most recent neuroimaging study with patients with delusional disorder (DD) with matched controls investigating working memory.

42. Kunert HJ, Norra C, Hoff P. Theories of delusional disorders. An update and review. Psychopathology. 2007;40(3):191-202. doi:10.1159/000100367.

43. Ibanez-Casas I, De Portugal E, Gonzalez N, McKenney KA, Haro JM, Usall J, et al. Deficits in executive and memory processes in delusional disorder: a casecontrol study. PLoS One. 2013;8(7):e67341. doi:10. 1371/journal.pone.0067341.

44.• Oflaz S, Akyuz F, Hamamci A, Firat Z, Keskinkilic C, Kilickesmez $\mathrm{O}$, et al. Working memory dysfunction in delusional disorders: an fMRI investigation. J Psychiatr Res. 2014;56:43-9. doi:10.1016/j.jpsychires.2014.04. 022.

The most recent neuroimaging study with patients with delusional disorder (DD) with matched controls investigating working memory.

45.• Freeman D, Garety P. Advances in understanding and treating persecutory delusions: a review. Soc Psychiatry Psychiatr Epidemiol. 2014;49(8):1179-89. doi:10. 1007/s00127-014-0928-7.

Introduced the six main proximal causal factors for delusions: worry thinking style, negative beliefs about the self, interpersonal sensitivity, sleep disturbances, anomalous internal experience, and reasoning biases.

46.• Garety P, Joyce E, Jolley S, Emsley R, Waller H, Kuipers $\mathrm{E}$, et al. Neuropsychological functioning and jumping to conclusions in delusions. Schizophr Res.

2013;150(2-3):570-4. doi:10.1016/j.schres.2013.08. 035.

Introduced the contributing cognitive factors to the occurrence of reasoning bias, specifically jumping to conclusion (JTC) among patients with delusions.

47.• Freeman D, Dunn G, Murray RM, Evans N, Lister R, Antley A, et al. How cannabis causes paranoia: using the intravenous administration of 9-tetrahydrocannabinol (THC) to identify key cognitive mechanisms leading to paranoia. Schizophr Bull. 2015;41(2):3919. doi:10.1093/schbul/sbu098.

A randomized, placebo-controlled study demonstrated that anomalous experiences and paranoia can be induced by intravenous administration of THC.

48. Bell V, Freeman D. A pilot trial of cognitive behavioural therapy for interpersonal sensitivity in individuals with persecutory delusions. J Behav Ther Exp Psychiatry. 2014;45(4):441-6. doi:10. 1016/j.jbtep.2014.06.001.

49. Freeman D, Lister R, Evans N. The use of intuitive and analytic reasoning styles by patients with persecutory delusions. J Behav Ther Exp Psychiatry.

2014;45(4):454-8. doi:10.1016/j.jbtep.2014.06.005.

50. Hepworth C, Startup H, Freeman D. Developing treatments of persistent persecutory delusions: the impact of an emotional processing and metacognitive awareness intervention. J Nerv Ment Dis. 2011;199(9):6538. doi:10.1097/NMD.0b013e318229cfa8. 
51. Myers E, Startup H, Freeman D. Cognitive behavioura treatment of insomnia in individuals with persistent persecutory delusions: a pilot trial. J Behav Ther Exp Psychiatry. 2011;42(3):330-6. doi:10.1016/j.jbtep. 2011.02.004.
52.

Ross K, Freeman D, Dunn G, Garety P. A randomized experimental investigation of reasoning training for people with delusions. Schizophr Bull. 2011;37(2):324-33. doi:10.1093/schbul/ sbn 165 . 